\title{
Santé et travail ou santé au travail
}

\section{Anne-Geneviève Bütikofer}

lic. iur., secrétaire générale de la FMH

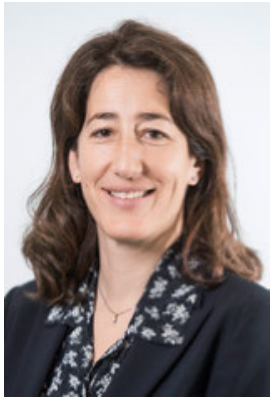

La santé au travail nous concerne toutes et tous, comme employeur, comme employé ou comme collègue. Le travail est bon pour la santé, mais les conditions de travail peuvent parfois y porter atteinte. La santé au travail nous concerne également comme individu, partenaire ou parent dans la mesure où elle influe directement sur notre vie privée. Peu importe la profession que l'on exerce, la fonction, la position, de nombreux facteurs peuvent toucher à l'intégrité physique ou psychique des personnes et engendrer diverses conséquences, ou risques psychosociaux, qu'il s'agisse de stress, d'épuisement professionnel, de mobbing, voire même de harcèlement.

Lévolution rapide du monde du travail, mais également la recrudescence des contraintes économiques ces dernières années, exigent de faire toujours plus avec toujours moins de ressources. Les pressions dans l'environnement de travail sont de nos jours de plus en plus fortes et souvent accompagnées de conséquences psychologiques. Et le domaine de travail de la santé n'est pas en reste.

Le Bureau international du Travail (BIT) et le Secrétariat d'Etat à l'économie (seco) rendent attentifs aux conditions de travail telles que notamment la surcharge, les exigences contradictoires et le manque de clarté sur la fonction à remplir, la non-participation des travailleurs aux décisions les concernant et leur absence d'influence sur le mode d'exécution de leur activité professionnelle, les changements organisationnels mal gérés et l'insécurité de l'emploi, l'inefficacité de la communication et le manque de soutien de la direction ou des collègues. Ces conditions sont susceptibles de déboucher sur des risques évoqués ci-dessus.

Notre société a évolué, et chacun d'entre nous souhaite mieux concilier vie personnelle et vie professionnelle. Le Secrétariat général de la FMH (SG), particulièrement attentif à ces questions, a initié en 2013 le projet de gestion de la santé en entreprise sanctionné par le label «Friendly Workspace» de la Fondation Promotion Santé Suisse, afin d'assurer un lieu de travail dans lequel travailleurs, cadres et direction travaillent ensemble à un processus d'amélioration continue qui vise à protéger et à promouvoir la santé, la sécurité et le bien-être de tous et la durabilité du lieu de travail. Même s'il ne prétend pas être parfait et même s'il n'est pas encore abouti, le projet nous a rendus beaucoup plus attentifs notamment à la santé, à la sécurité et au bien-être dans l'environnement de travail psychosocial, y compris l'organisation du travail et la culture sur le lieu de travail.

Plusieurs articles et éditoriaux du département Santé publique dans le BMS (2015;96[25]:899 / 2016;97[5]:167) ont, au cours des dernières années, rappelé par ailleurs que les médecins sont soumis à un stress psychosocial important dans leur activité quotidienne et que les changements dans leurs conditions de travail se traduisent souvent par de l'épuisement professionnel, alors que leur santé est importante. A cet égard, la FMH, sous la houlette du département Santé publique, va poursuivre son engagement dans ce domaine et continuera d'organiser les symposiums «Des médecins en bonne santé» afin de contribuer à la promotion et à la préservation de la santé des médecins.

Pour un jeune médecin, un médecin avec une longue expérience professionnelle ou un collaborateur du SG de la FMH, travailler, collaborer, trouver de nouvelles solutions et atteindre ses objectifs est une source de plaisir et d'énergie. Aussi, au-delà d'une simple obligation légale, il en va de notre responsabilité sociale à tous, dès lors que l'on travaille avec des collègues, des employés ou des patients, de prendre toutes les mesures nécessaires pour protéger l'intégrité personnelle, prévenir les risques psychosociaux et par là même maintenir le plaisir de travailler et la confiance vis-àvis de ses collègues de travail, encourager la croissance des performances et faire usage des aptitudes et des chances professionnelles, en résumé éviter tout impact néfaste sur le bien-être professionnel et privé des uns et des autres.

En espérant que ces propos puissent avoir une influence positive sur les résolutions de fin d'année, je souhaite à toutes les lectrices et tous les lecteurs de belles et reposantes fêtes de fin d'année ainsi qu'un passage serein vers l'an neuf. 